\title{
Utilizing Correlative Imaging Approaches with ToF-SIMS Expands Our Biochemical Interpretation Abilities Across Biological Kingdoms
}

Christopher Anderton ${ }^{1}$, Arunima Bhattacharjee ${ }^{1}$, Jocelyn Richardson ${ }^{2}$, Tina Anger ${ }^{3}$, Odeta Qafoku ${ }^{1}$, Dušan Veličković ${ }^{1}$, Michael Taylor ${ }^{1}$, Zihua Zhu ${ }^{1}$, Daniel Graham ${ }^{4}$, Lara Gamble ${ }^{3}$, Kirsten Hofmockel ${ }^{1}$ and Janet Jansson ${ }^{1}$

${ }^{1}$ Pacific Northwest National Lab, Richland, Washington, United States, ${ }^{2}$ Stanford Synchrotron Radiation Lightsource, Menlo Park, California, United States, ${ }^{3}$ University of Washington, Seattle, Washington, United States, ${ }^{4}$ University of Washington-Department of Bioengineering, Seattle, Washington, United States

The Environmental Molecular Sciences Laboratory (EMSL) is a U.S. Department of Energy National User Facility, located on the campus of Pacific Northwest National Laboratory, which houses a range of capabilities dedicated to addressing some of the most critical challenges facing our nation and the world. Within its suite of capabilities, EMSL houses a number of imaging tools, including time-of-flight secondary ion mass spectrometry (ToF-SIMS; IONTOF V) and other mass spectrometry imaging (MSI) systems. We recently used ToF-SIMS to understand processes that govern fungal mycelial bridging of nutrient sources within simulated soil microenvironments. Here, we inoculated Fusarium chlamydosporum into soil micromodels that concurrently simulate the porosity and mineralogy of soil, where ToF-SIMS demonstrated cation enrichment and micronutrient translocation from minerals by fungal hyphae. These results were correlated with data acquired using X-ray near edge absorption analysis of fungal growth in mineral doped micromodels, which showed mineral speciation as result of hyphal uptake of micronutrients from minerals. Furthermore, ToF-SIMS and SEM/EDX enabled us to observe how fungi would biosense specific mineral types within the micromodels. In other work done in conjunction with the University of Washington, we utilized their unique ion bunching, tandem MS capable, gas cluster primary ion beam ToF-based SIMS instrument (J105-3D Chemical Imager; Ionoptika Ltd.), in combination with our bulk lipidomics capabilities (e.g., liquid chromatography tandem mass spectrometry), to show that we could simultaneously localize lipids, with high confidence in their identifications, within flatworms (Phagocata gracilis). A follow up study confirmed their localization and identifications using the ultrahigh mass resolution matrix-assisted laser desorption/ionization (MALDI)MSI system housed in EMSL. These results and more demonstrate the value of ToF-SIMS in increasing our ability to interpret complex biological processes, particularly when used in coordination with other imaging modalities.

\section{References}

Tina B.Angerer, Neil Chakravarty, Michael J.Taylor, Carrie D. Nicora, Daniel J.Graham, Christopher R.Anderton, Eric H.Chudler, Lara J. Gamble Biochimica et Biophysica Acta (BBA) - Molecular and Cell Biology of Lipids 2019 1864, 733

Tina B. Angerer, Dusan Velickovic, Carrie D. Nicora, Jennifer E. Kyle, Daniel J. Graham, Christopher Anderton, and Lara J. Gamble

Analytical Chemistry 201991 (23), 15073 